\title{
Homology modeling and Molecular docking studies of AS1 (Anthranilate synthase component I (TrpE)) model of Mycobacterium tuberculosis
}

\author{
Naresh kumar K, \\ Department of Biotechnology, Institute of Biological Science, Vijayawada, Andhra Pradesh -520010, India. \\ Suresh Kumar C, \\ Department of Biochemistry, Sri Krishnadevaraya University, Anantapur Andhra Pradesh - 515 003, India \\ Subba Rao D, \\ Department of Chemical Engineering, Jawaharlal Nehru Technological University, Anantapur Andhra Pradesh 515001 \\ India.
}

\begin{abstract}
The emergence of multi-drug resistant (MDR) strains of Mycobacterium tuberculosis is the main reason why tuberculosis (TB) continues to be a major health problem worldwide. It is urgent to discover novel antimycobacterial agents based on new drug targets for the treatment of TB, especially MDR-TB. Tryptophan biosynthetic pathway, which is essential for the survival of M. tuberculosis and meanwhile absent in mammals, provides potential anti-TB drug targets. One of the promising drug targets in this pathway is anthranilate synthase component I (TrpE), whose role is to catalyze the conversion of chorismate to anthranilate using ammonia as amino source. Anthranilate synthase is an interesting target enzyme for antimicrobial activity due to its presence in microorganisms for the synthesis of the essential amino acid tryptophan. In the present study three compounds Cannabigerolic acid, cannabinolic acid and adhumulone from Cannabis sativa have been used for insilio docking studies. Inhibitory studies (invitro) of these compounds against Microorganism have reported earlier. Our approach is to find out the compounds inhibiting the AS1 of MTB by insilico docking and also find out compounds having similar pharmacophore characters from ZINC database so that those compounds can be procured of synthesized in laboratory and used for AS1 inhibitor studies. This study shows that AS can be used as a target enzyme to investigate the mode of action of our compounds in MTB.
\end{abstract}

Key words: Mycobacterium tuberculosis, AS1 MTB, Homology modeling, Docking.

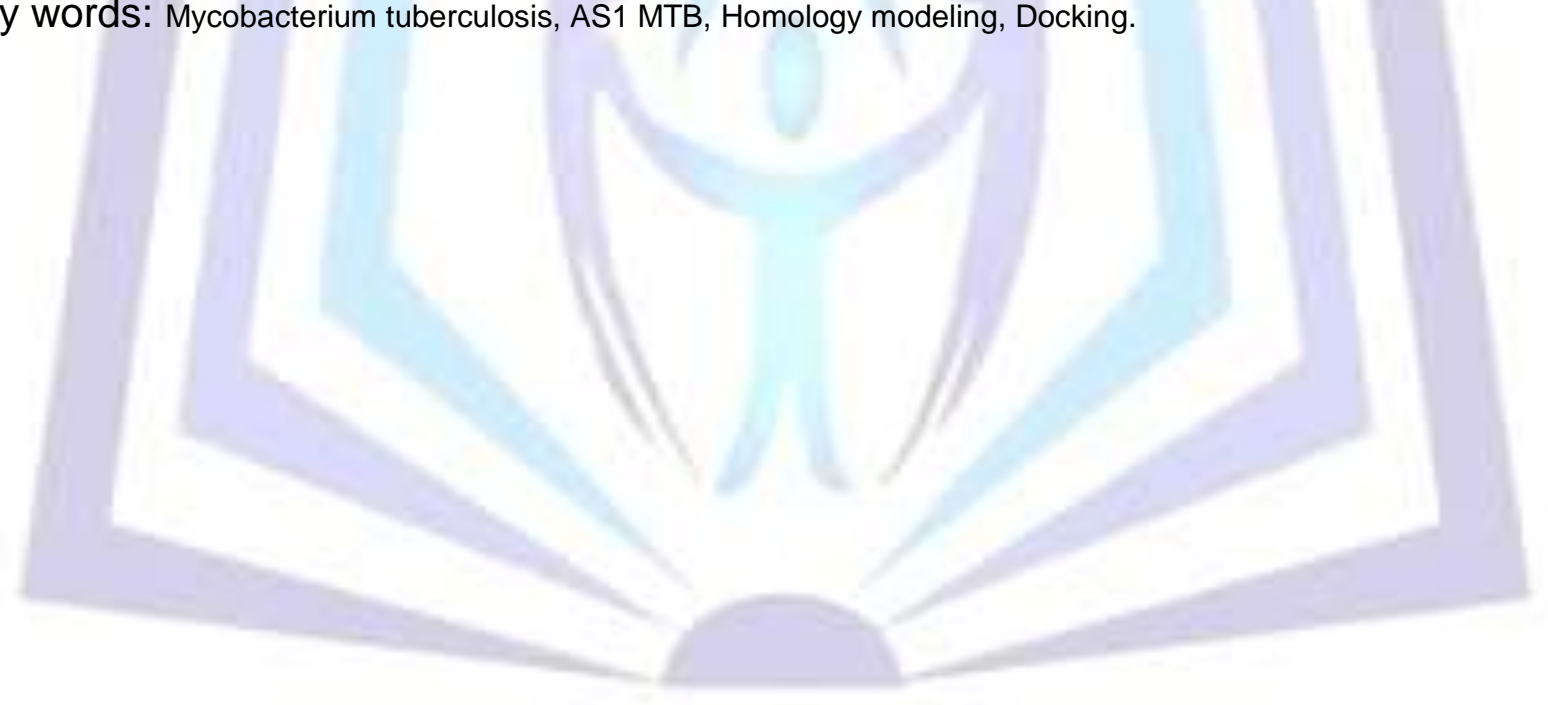

\section{Council for Innovative Research}

Peer Review Research Publishing System

Journal: INTERNATIONAL JOURNAL OF COMPUTERS \& TECHNOLOGY

Vol 10, No 1 


\section{INTRODUCTION}

Tuberculosis (TB), caused by Mycobacterium tuberculosis (M. tuberculosis), remains one of the most devastating global diseases. About one-third of the world population is infected with M. tuberculosis and almost 5000 people die from TB everyday (Corbett et al., 2003). Recently, long-term antibiotic therapy has led to the emergence and wide spread of multi-drug resistant (MDR) M. tuberculosis strains. Over 300,000 new cases of MDR-TB occur annually worldwide (World Health Organization 2004) and meanwhile the treatment for MDR-TB is becoming less effective than ever. Therefore, discovery of novel anti-mycobacterial agents based on new drug targets is an urgent task to combat TB, especially MDR-TB.

Anthranilate synthase (AS 1, EC 4.1.3.27), a member of tryptophan biosynthetic pathway, catalyzes the formation of anthranilate from chorismate and ammonia (or glutamine), which is the first committed step branching from the common aromatic amino acid (shikimate) pathway toward the biosynthesis of I-tryptophan. AS from microorganisms are made up of synthase component (TrpE) and glutamine amidotransferase component (TrpG), namely component I and component II, respectively (Morollo and Eck 2001; Spraggon et al., 2001; Knochel et al., 1999). TrpE is able to catalyze the synthesis of anthranilate with ammonia as the source of nitrogen atom independently (Knochel et al., 1999; Ito et al., 1969; Hankins and Mills 1976).

TrpE : Chorismate + ammonia ---------- $\rightarrow$ anthranilate + pyruvate +water

Moreover, the l-tryptophan binding site of AS is located on TrpE component (Morollo and Eck 2001; Knochel et al., 1999; Zalkin and Kling 1968), therefore the inhibition of TrpE activity might cause the failure of tryptophan biosynthesis. In summary, TrpE is a promising drug target for the design of novel anti-TB drugs.

The importance of computational approaches in designing selective ligands has become indispensable in the current drug design and discovery protocols. Homology modeling involves taking a known sequence with an unknown structure (target) and mapping it against a known structure (template) of one or several homologous proteins. The homology modeling is mainly based on the structural conservation of the framework regions between the members of a protein family. Proteins from different sources and sometimes diverse biological functions can have similar sequences. In general the process of homology modeling involves four sequential steps such as template selection, target-template alignment, model construction, and model assessment.

\section{MATERIALS AND METHODS}

\subsection{Homology modeling 3D model building}

Homology modeling, also known as comparative modeling refers to constructing an atomic-resolution model of the "target" protein from its amino acid sequence and an experimental three-dimensional structure of a related homologous protein (the "template"). Homology modeling relies on the identification of one or more known protein structures likely to resemble the structure of the query sequence, and on the production of an alignment that maps residues in the query sequence to residues in the template sequence. The sequence alignment and template structure are then used to produce a structural model of the target. Because protein structures are more conserved than DNA sequences, detectable levels of sequence similarity usually imply significant structural similarity.

Suppose you want to know the 3D structure of a target protein that has not been solved empirically by $\mathrm{X}$-ray crystallography or NMR. You have only the sequence. If an empirically determined 3D structure is available for a sufficiently similar protein (50\% or better sequence identity would be good), you can use software that arranges the backbone of your sequence identically to this template. This is called "homology modeling". It is, at best, moderately accurate for the positions of alpha carbons in the 3D structure, in regions where the sequence identity is high. It is inaccurate for the details of side chain positions and for inserted loops with no matching sequence in the solved structure.

The query sequence from AS1 of mtb was submitted to domain fishing server Signal for prediction. The predicted domain was searched to find out the related protein structure to be used as a template by the BLAST (Basic Local Alignment Search Tool) program against PDB (Protein Databank). Sequence that showed maximum identity with high score and less e-value was aligned and 1I7Q of PDB structure of S. marcescens was used as a reference structure to build a 3D model for. The co-ordinates for the structurally conserved regions (SCRs) for AS1 of mtb were assigned from the template using multiple sequence alignment, based on the Needleman-Wunsch algorithm.

The final structure obtained was analyzed by Ramachandran's map using PROCHECK (Programs to check the Stereo chemical Quality of Protein Structures) and environment profile using ERRAT graph (Structure Evaluation server). This model was used for the identification of active site and for docking of the substrate with the enzyme.

\subsection{Active site Identification}

Active site of AS1 model of MTB was identified using CASTp. A new program, CASTp, for automatically locating and measuring protein pockets and cavities, is based on precise computational geometry methods, including alpha shape and discrete flow theory. CASTp identifies and measures pockets and pocket mouth openings, as 
well as cavities. The program specifies the atoms lining pockets, pocket openings, and buried cavities; the volume and area of pockets and cavities; and the area and circumference of mouth openings.

\subsection{Docking method}

Docking was carried out using GOLD (Genetic Optimization of Ligand Docking) software which is based on genetic algorithm (GA). This method allows as partial flexibility of protein and full flexibility of ligand. The compounds are docked to the active site of the AS1 model of MTB. The interaction of these compounds with the active site residues are thoroughly studied using molecular mechanics calculations. After docking, the individual binding poses of each ligand were observed and their interactions with the protein were studied. The best and most energetically favorable conformation of each ligand was selected.

\subsubsection{Docking Studies.}

Protein preparation: The crystal structure of MTB AS1 is not yet available. So we have modeled the AS1 of MTB by using homology modeling. Hereafter we use the name of our target as AS1 model. For homology modeling purpose we have used 117q PDB ID of S. marcescens Anthranilate synthase as template.

\subsubsection{Gold Score fitness function}

Gold Score performs a force field based scoring function and is made up of four components: 1. Protein-ligand hydrogen bond energy (external H-bond); 2. Protein-ligand vander Waals energy (external vdw); 3. Ligand internal vander Waals energy (internal vdw); 4. Ligand intramolecular hydrogen bond energy (internal- $\mathrm{H}$ - bond). The external vdw score is multiplied by a factor of 1.375 when the total fitness score is computed. This is an empirical correction to encourage protein-ligand hydrophobic contact. The fitness function has been optimized for the prediction of ligand binding positions.

GoldScore $=S$ (hb_ext $)+S\left(v d w \_e x t\right)+S$ (hb_int $)+S$ (vdw_int $)$

Where $S$ (hb_ext) is the protein-ligand hydrogen bond score, $S$ (vdw_ext) is the protein-ligand van der Waals score, $S$ (hb_int) is the score from intramolecular hydrogen bond in the ligand and S (vdw_int) is the score from intramolecular strain in the ligand.

For each independent genetic algorithm (GA) run, a maximum of 100000 operations were performed on a populat ion of 5 islands, each of 100 individuals. Operator weights for crossover, mutation and migration were set to 95, 95 and 10 respectively. To allow for development of close contacts and poor hydrogen bonds occurring at the beginning of each GA run, the initial external Vander Waals ( $\mathrm{vdw}$ ) energies were cut off at 2.5k, where $\mathrm{k}$ is the depth of the vdw well between atoms $i$ and $j$, and the maximum distance between a donor atom and a fitting point was set to $4 \AA$. Ring flipping and internal hydrogen bonds were allowed. The dockings were terminated after 25 runs for each of the inhibitors.

Various reports shown that Anthranlate synthase can be used as drug target and many researchers have designed inhibitors for Anthranilate synthase, but all are synthetic. In the present study we have used the natural compounds like cannabigerolic acid, cannabinolic acid, adhumulone extracted from plants. We have used these compounds as ligands and performed docking with AS1 model of MTB using GOLD software. Comparitively we docked the inhibitors like 4 methoxy benzoate and 2 methoxy benzoate also with AS1 model.

\subsubsection{ZINK}

The ZINC Database contains commercially available compounds for structure based virtual screening. It currently has about 20 million compounds that can simply be purchased. It is provided in ready-to-dock, 3D formats with molecules represented in biologically relevant forms. It is available in subsets for general screening as well as target-, chemotype- and vendor-focused subsets. ZINC is free for everyone to use and download at the website zinc.docking.org. This database and service is provided by the Shoichet Laboratory in the Department of Pharmaceutical Chemistry at the University of California San Francisco (UCSF). To cite ZINC, please reference: Irwin and Shoichet, J. Chem. Inf. Model. 2005; 45(1):177-82 PDF, DOI. We thank NIGMS for financial support (GM71896).

The purpose of this search is the compounds used here are naturally available from plant source. Availability of these compounds in large scale is practically not feasible. So our approach is to search for compounds having similar pharmacophore features in ZINC database so that after docking they can directly purchased from the respective vendor and used for inhibitory studies on AS1 of MTB. 
2.4 Different Natural compounds were checked for the docking with protein MTB AS1 as following

\begin{tabular}{|l|l|l|l|}
\hline $\begin{array}{l}\text { S } \\
n\end{array}$ & $\begin{array}{l}\text { Name of the } \\
\text { compounds }\end{array}$ & $\begin{array}{l}\text { Pubche } \\
\mathrm{m} \text { ID }\end{array}$ & SMILES \\
\hline 1 & $\begin{array}{l}\text { Cannabigerolic } \\
\text { acid }\end{array}$ & $\begin{array}{l}644999 \\
9\end{array}$ & $\begin{array}{l}\mathrm{CCCCCC} 1=\mathrm{CC}(=\mathrm{C}(\mathrm{C}(=\mathrm{C} 1 \mathrm{C}(=\mathrm{O}) \\
\mathrm{O}) \mathrm{O}) \mathrm{CC}=\mathrm{C}(\mathrm{C}) \mathrm{CCC}=\mathrm{C}(\mathrm{C}) \mathrm{C}) \mathrm{O}\end{array}$ \\
\hline 2 & cannabinolic acid & 308199 & $\begin{array}{l}\mathrm{CCCCCC} 1=\mathrm{CC}=\mathrm{C}(\mathrm{C} 3=\mathrm{C}(\mathrm{C}=\mathrm{C} \\
\mathrm{C}(=\mathrm{C} 3) \mathrm{C}) \mathrm{C}(\mathrm{O} 2)(\mathrm{C}) \mathrm{C}) \mathrm{C}(=\mathrm{C} 1 \mathrm{C}(= \\
\mathrm{O}) \mathrm{O}) \mathrm{O}\end{array}$ \\
\hline 3 & 0 & $\begin{array}{l}\mathrm{CCC}(\mathrm{C}) \mathrm{C}(=\mathrm{C} 1 \mathrm{C}(=\mathrm{O}) \mathrm{C}(\mathrm{C}(=\mathrm{O}) \mathrm{C}( \\
\mathrm{C} 1=\mathrm{O})(\mathrm{CC}=\mathrm{C}(\mathrm{C}) \mathrm{C}) \mathrm{O}) \mathrm{CC}=\mathrm{C}(\mathrm{C}) \mathrm{C} \\
\mathrm{O}\end{array}$ \\
\hline
\end{tabular}

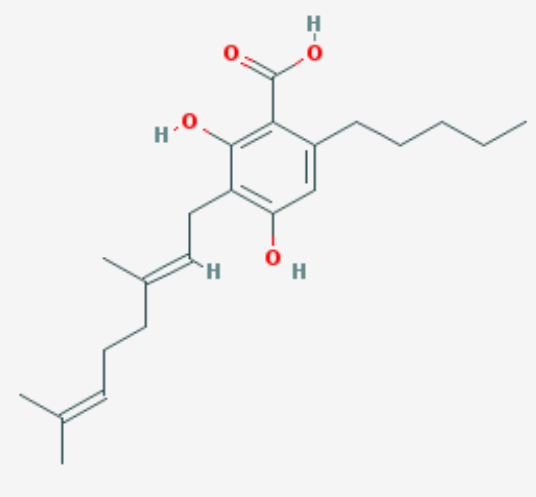

Fig1A: Cannabigerolic acid

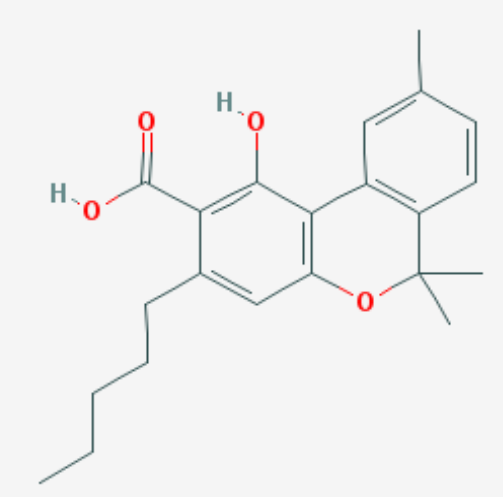

Fig 1B: Cannabinolic acid

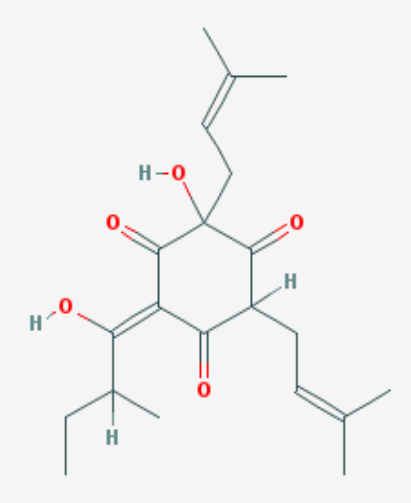

Fig 1C: Adhumulone 
2.5 Different Synthetic compounds were checked for the docking with protein MTB AS1 as following

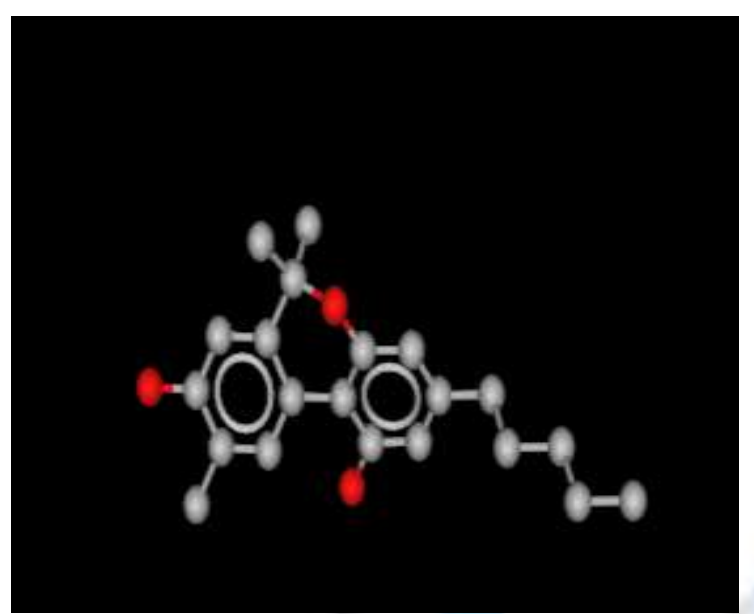

Fig 2a: ZINC43059689

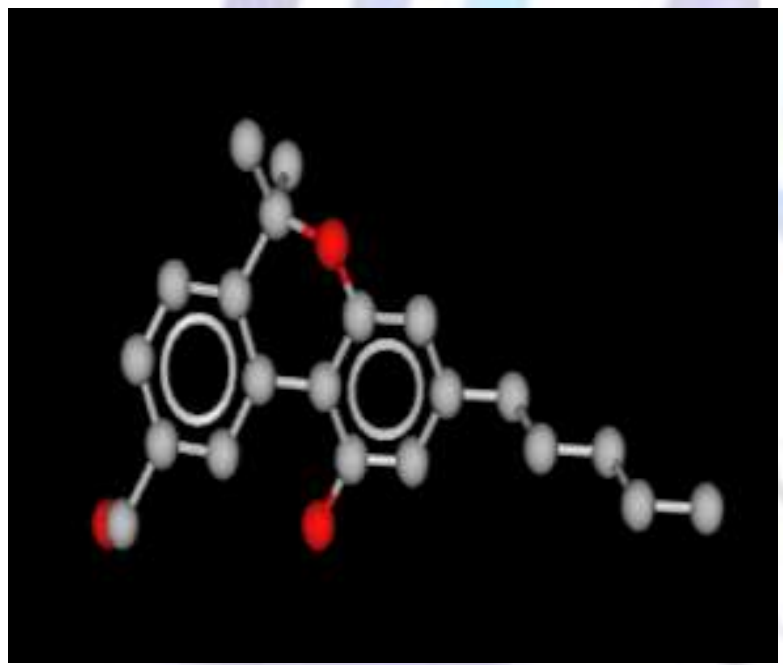

Fig 2c: ZINC38139497

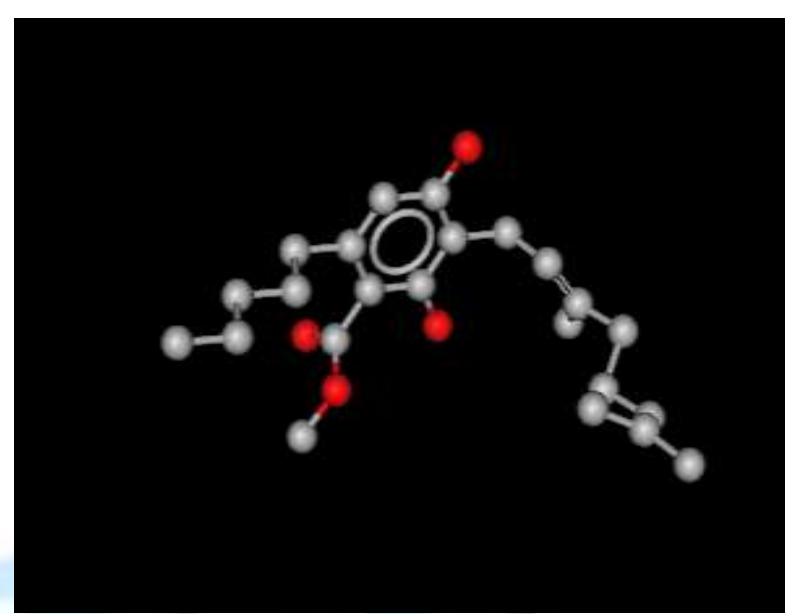

Fig 2b: ZINC40973021

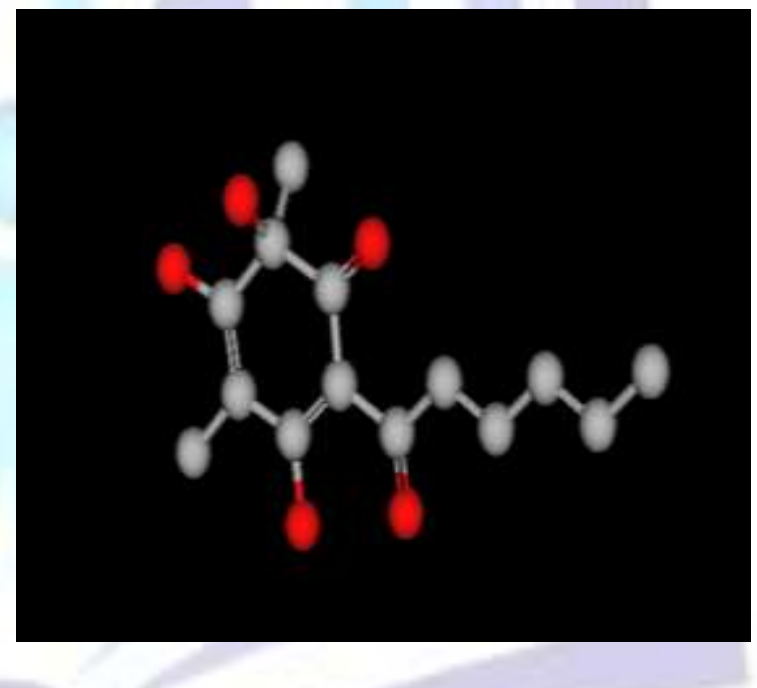

Fig 2d: ZINC13765599 


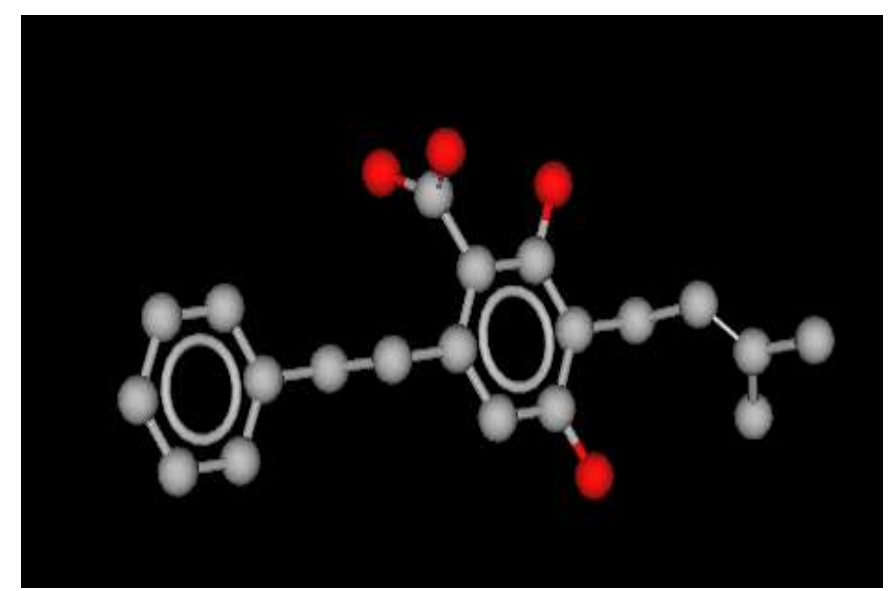

Fig 2e: ZINC13340592

\section{RESULTS AND DISCUSSION}

\subsection{Homology Modeling of MTB AS1 Protein}

A high level of sequence identity should guarantee more accurate alignment between the target sequence and template structure. In the results of BLAST search against PDB, 117Q of PDB structure of S. marcescens has a high level of sequence identity and the identity of the reference protein with the MTB AS1 are $48 \%$.

Sequences were aligned using the CLUSTAL W (1.82) multiple sequence alignment software "*” indicates that the amino acid residues in that column are ident ical in all sequences of the alignment ":" indicates that conserved residues have been ident ified amongst the sequences in the alignment "." indicates that semi-conserved substitutions have been observed amongst the sequences in the alignment. Residues coloured with red and the active site residues, which are conserved in serratia and mtb proteins figure 3 .

MTB --MHADLAATTSREDFR--LLAAEHRVVPVTRKVLADSETPLSAYRKLAANRPGTFLLES 56

117Q_C MNTKPQLTLLKVQASYRGDPTTLFHQLCGARPATLLLESAEINDKQNLQSLLVIDSALRI 60 $: *^{*}: .::^{*} \quad: *^{*}: . .^{*} \ldots:::^{*}: \quad$ *.

MTB AENGRSWSRWSFIGAGAPTALTVREGQ--AVWLGAVPK--------DAPTGGDP-LRAL 104

117Q_C TALGHTVSVQALTANGPALLPLLDEALPPEVRNQARPNGRELTFPAIDAVQDEDARLRSL 120

MTB QVTLELLATADRQSEPGLPPLSGGMVGFFAYDMVRRLERLPERAVDDLCLPDMLLLLATD 164

1I7Q_C SVFDALRTILTLVDSPADEREAVMLGGLFAYDLVAGFENLPALRQDQRC-PDFCFYLAET 179

MTB VAAVDHHEGTITLIANAVNWNGTDERVDWAYDDAVARLDVMTAALGQPLPSTVATFSRPE 224

117Q_C LLVLDHQRGSARLQASVFSEQASEAQR------LQHRLEQLQAELQQP-PQPIPHQKLEN 232 $: .{ }^{* *} . *^{*}:{ }^{*} \ldots . . . .:: \quad{ }^{* *}::^{* * * *} \ldots . . .:$

MTB PRHRAQRTVEEYGAIVEYLVDQIAAGEAFQVVPSQRFEMDTDVDPIDVYRILRVTNPSPY 284

117Q_C MQLSCNQSDEEYGAVVSELQEAIRQGEIFQVVPSRRFSLPCPA-PLGPYQTLKDNNPSPY 291

MTB MYLLQVPNSDGAVDFSIVGSSPEALVTVHEG--WATTHPIAGTRWRGRTDDE------DV 336

117Q_C MFFMQDD------DFTLFGASPESALKYDAGNRQIEIYPIAGTRPRGRRADGSLDLDLDS 345

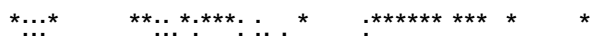

MTB LLEKELLADDKERAEHLMLVDLGRNDLGRVCTPGTVRVEDYSHIERYSHVMHLVSTVTGK 396

117Q_C RIELEMRTDHKELAEHLMLVDLARNDLARICQAGSRYVADLTKVDRYSFVMHLVSRVVGT 405

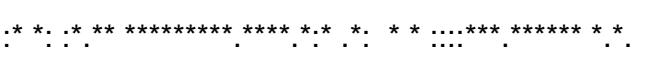




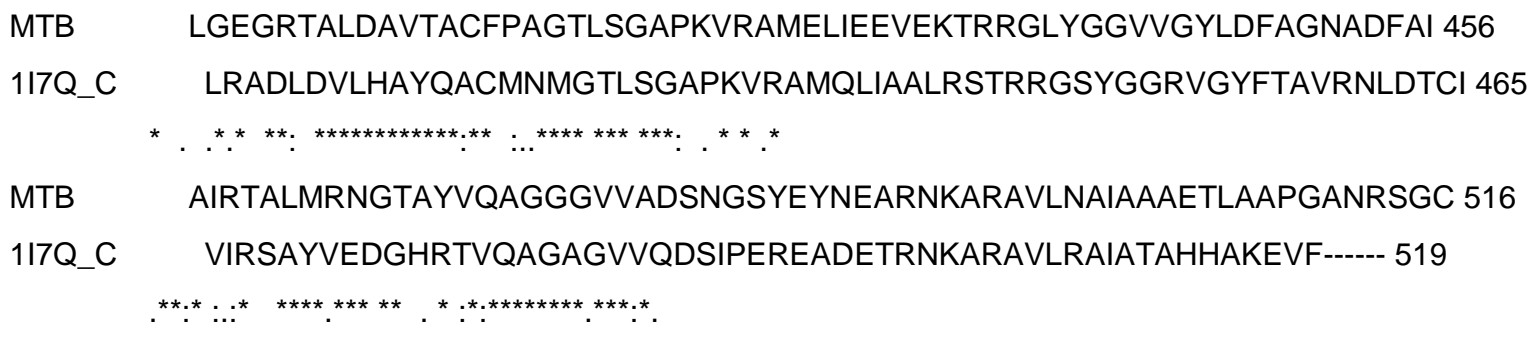

Figure 3: Clustal pair wise alignment,

In the following study, we have chosen $1 \mathrm{I7Q}$ of PDB structure of $\mathrm{S}$. marcescens as a reference structure for modeling domain. Coordinates from the reference protein 1 I7Q of PDB structure of S. marcescens to the SCRs, structurally variable regions (SVRs), $\mathrm{N}$-termini and $\mathrm{C}$-termini were assigned to the target sequence based on the satisfaction of spatial restraints. In the modeller we will get a 20 PDB out of which we select a least energy. The energy unit will be in kilo joule. All side chains of the model protein were set by rotamers. The final stable structure of the MTB AS1 protein obtained is shown in Figure 4. By the help of SPDBV.

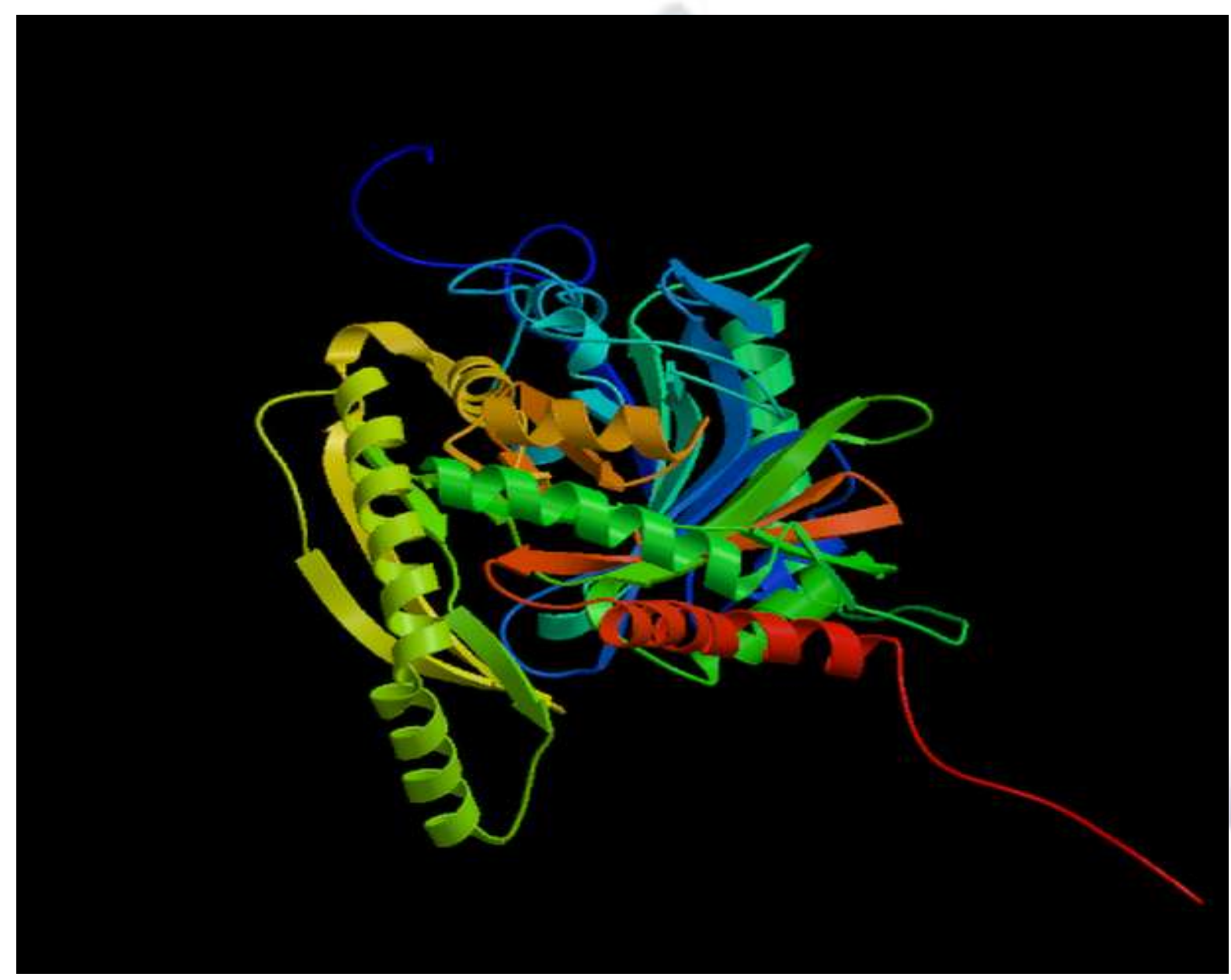

Figure 4: Structure of the MTB AS1 protein

\subsection{Active site Identification of MTB AS1}

After the final model was built, the possible binding sites of MTB AS1 was searched based on the structural comparison of template and the model build and also with CASTP server and was shown in Figure 5. Since, MTB AS1 from Human and the 1I7Q of PDB structure of S. marcescens are well conserved in both sequence and structure; their biological function should be identical. In fact from the structure-structure comparison of template, final refined model of MTB AS1 domain using 34 SPDBV program. 
Table 1 : Active Site Residues

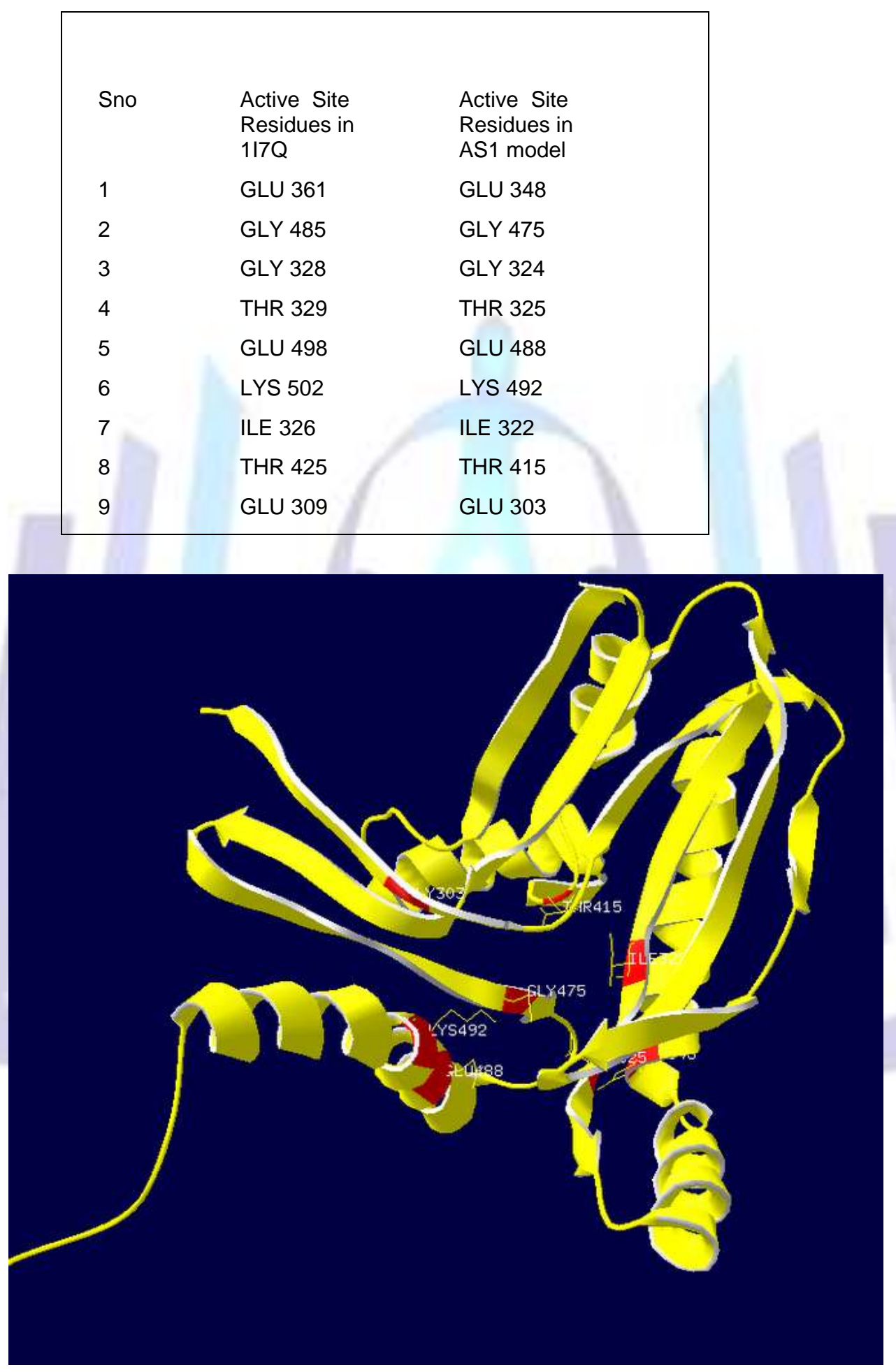

Fig: xx MTB AS 1 model showing Active site residues 


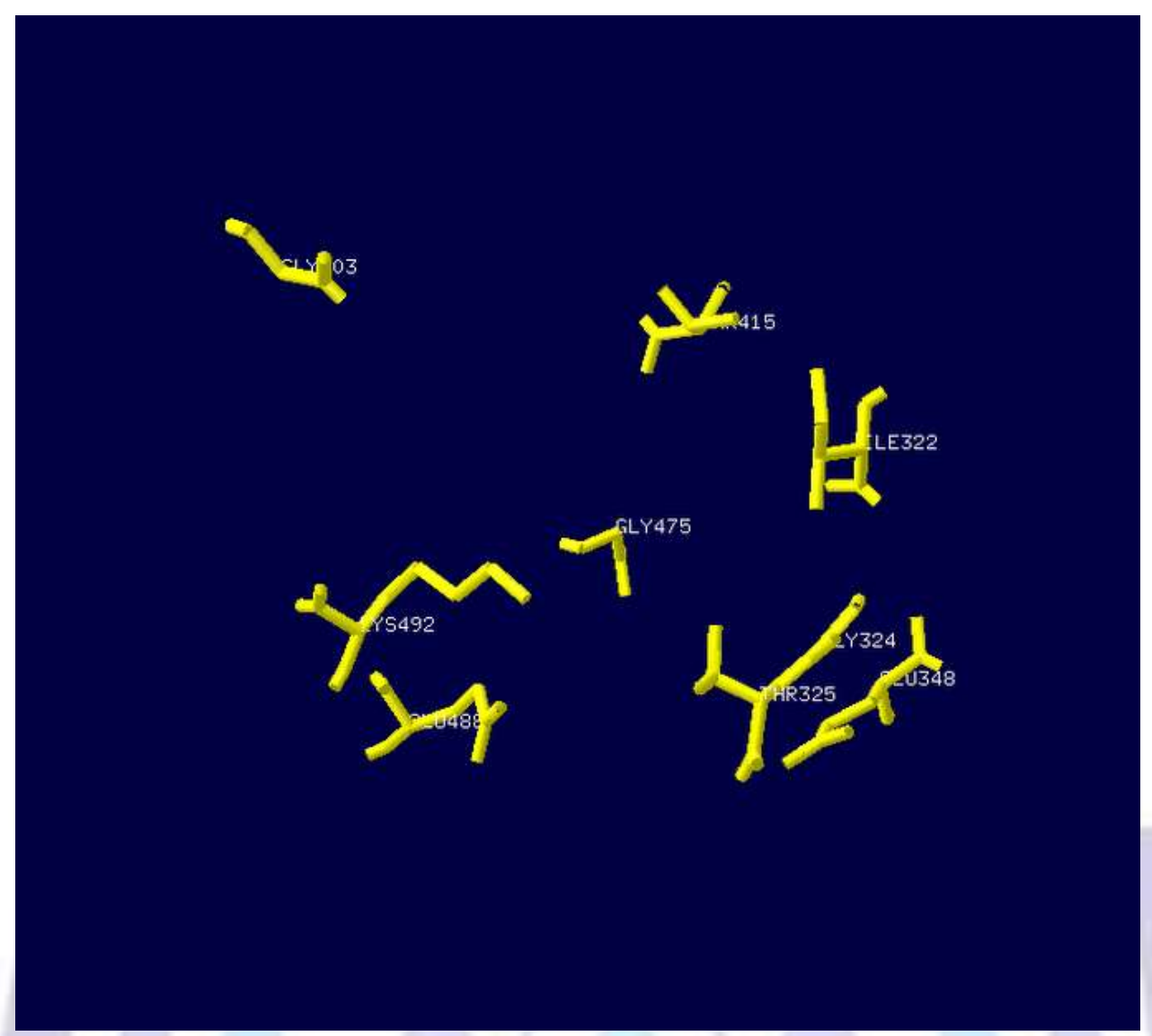

Fig: 5 Active Site Residues of MTB AS1 model

\subsection{Docking of inhibitors with the active site of protein MTB AS1}

\subsubsection{Natural copmponds}

Fitness $\mathbf{S}$ (hb_ext) $\mathbf{S}\left(v d w \_e x t\right) S(h b$ int) $\mathbf{S}$ (int) time File name

Ligand name

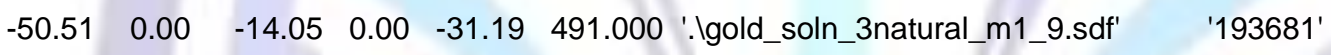

$\begin{array}{llllll}-157.64 & 0.02 & -97.43 & 0.00 & -23.70 & 324.000\end{array}$ '.lgold_soln_3natural_m2_2.sdf' '3081990'

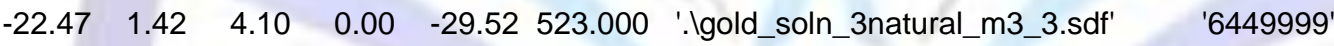

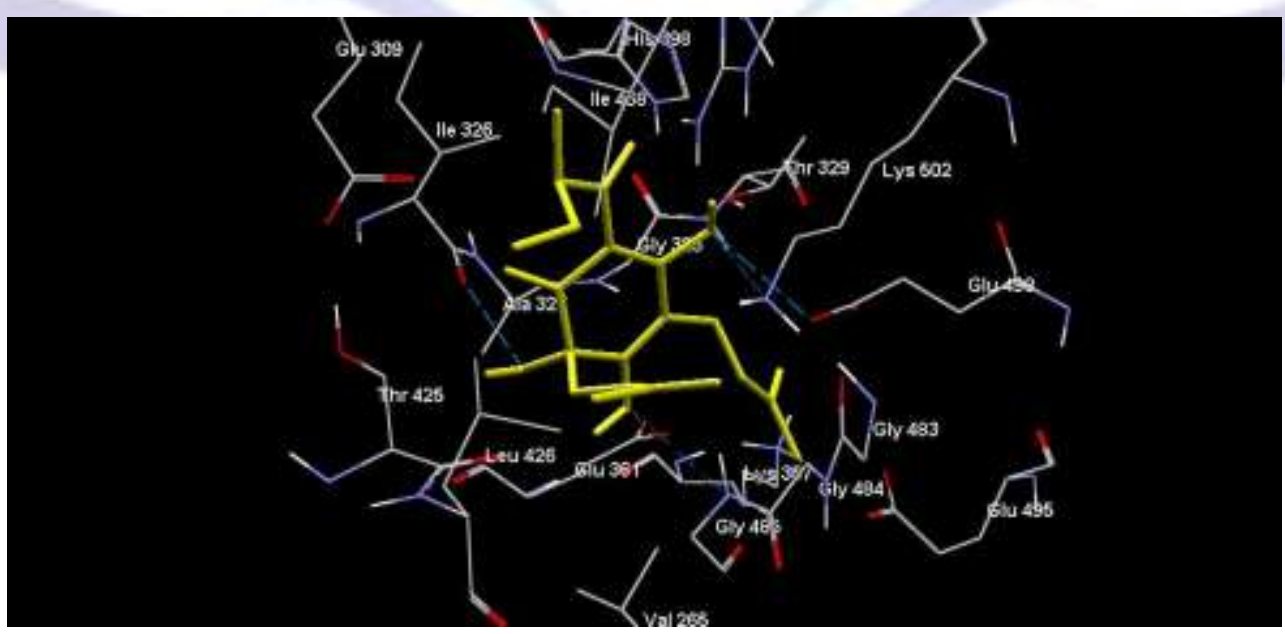

Fig 6a: Docking of molecule 1 pose 9 of 193681 - adhumulone

Interacting residues glu 361, Lys 357, Gly 485, thr 329, glu 498, Lys 502 


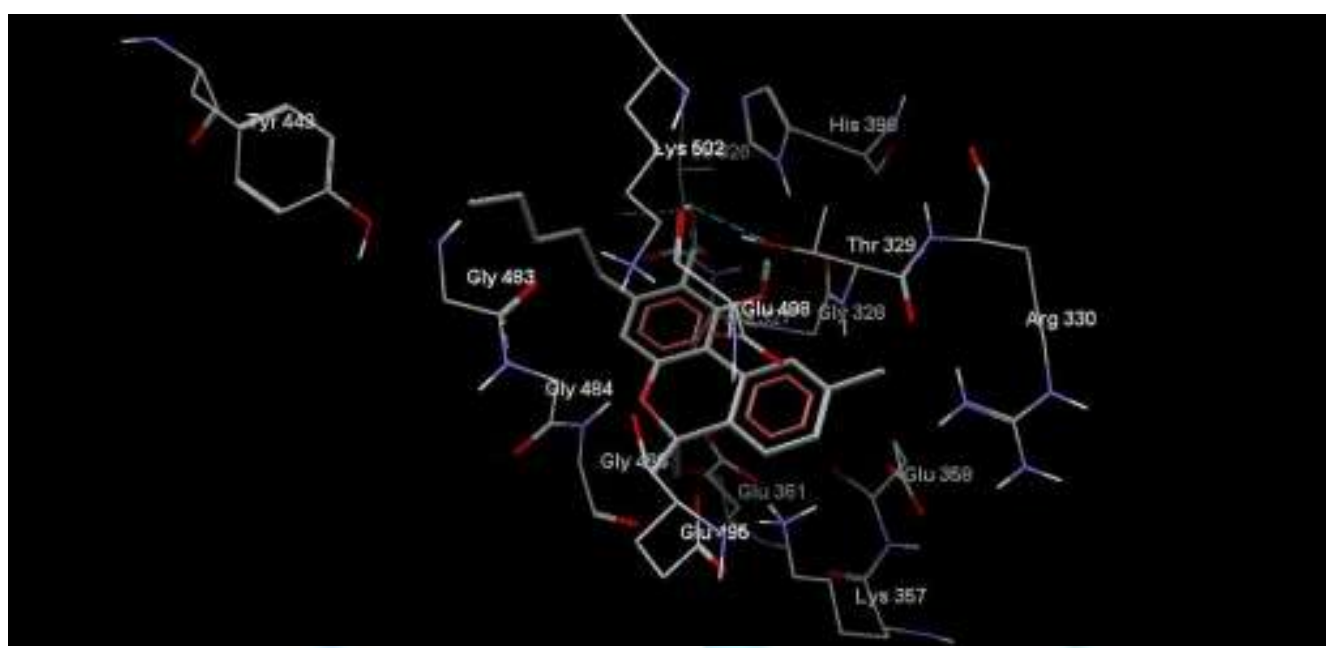

Fig 6b: Docking of molecule 2 pose 2 of 3081990 - cannabinolic acid Interacting residues - Lys 502, Thr 329, Gly 328, Glu 498, Glu 361

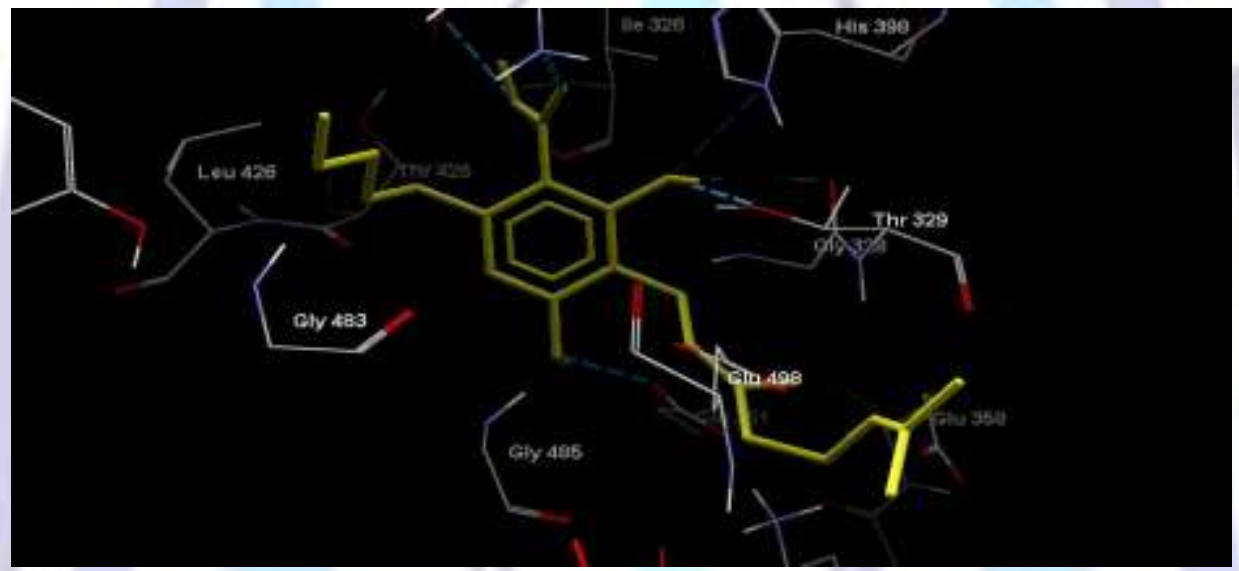

Fig 6c: Docking of molecule 3 pose 3 of - 6449999 - cannabigirolic acid Interacting residues - Thr 425, Glu 361, glu 498, thr 329, gly 328, ile 326

In ZINC searching for adhumulone similar compounds we have got 40 compounds synthetic, In zinc searching for cannabigerolic acid similar compounds we have got 22 compounds, In zinc searching for cannabinolic acid similar compounds we have got 19 compounds. For 3 compounds from plant we could search total 81 compounds from zinc database, finally top 5 synthetic compounds taken for the docking studies.

\subsubsection{Top 5 Synthetic similar compounds}

\begin{tabular}{|c|c|c|c|c|c|c|}
\hline $\begin{array}{l}\text { Fitness } \\
\text { name }\end{array}$ & \multicolumn{2}{|c|}{$S\left(h b \_e x t\right) S$} & $S\left(v d w \_e x t\right)$ & S(hb_int) & S(int) & time File name \\
\hline $\begin{array}{l}-31.80 \\
\text { 'ZINC13340 }\end{array}$ & $\begin{array}{r}0.00 \\
0592\end{array}$ & -9.25 & 0.00 & -19.08 & 137.000 & '.Igold_soln_6449999 like_m17_1.sdf' \\
\hline $\begin{array}{lr}-88.14 & 0 \\
\text { 'ZINC13765 }\end{array}$ & $\begin{array}{l}0.00 \\
5599\end{array}$ & -64.10 & 0.00 & -316.16 & 167.000 & '.Igold_soln_3081990 like_m17_4.sdf' \\
\hline $\begin{array}{l}-13.88 \\
\text { 'ZINC38139 }\end{array}$ & $\begin{array}{r}0.00 \\
9497\end{array}$ & 21.04 & 0.00 & -15.04 & 157.000 & '.Igold_soln_193681 like_m2_7.sdf' \\
\hline $\begin{array}{l}-31.81 \\
\text { 'ZINC40973 }\end{array}$ & $\begin{array}{r}0.00 \\
3021\end{array}$ & 4.39 & 0.00 & -37.852 & 267.000 & '.Igold_soln_6449999 like_m4_2.sdf' \\
\hline $\begin{array}{l}-37.52 \\
\text { ZINC43059 }\end{array}$ & $\begin{array}{r}0.03 \\
9689\end{array}$ & -27.30 & 0.00 & -939.74 & 141.001 & '.Igold_soln_3081990 like_m15_5.sdf' \\
\hline
\end{tabular}




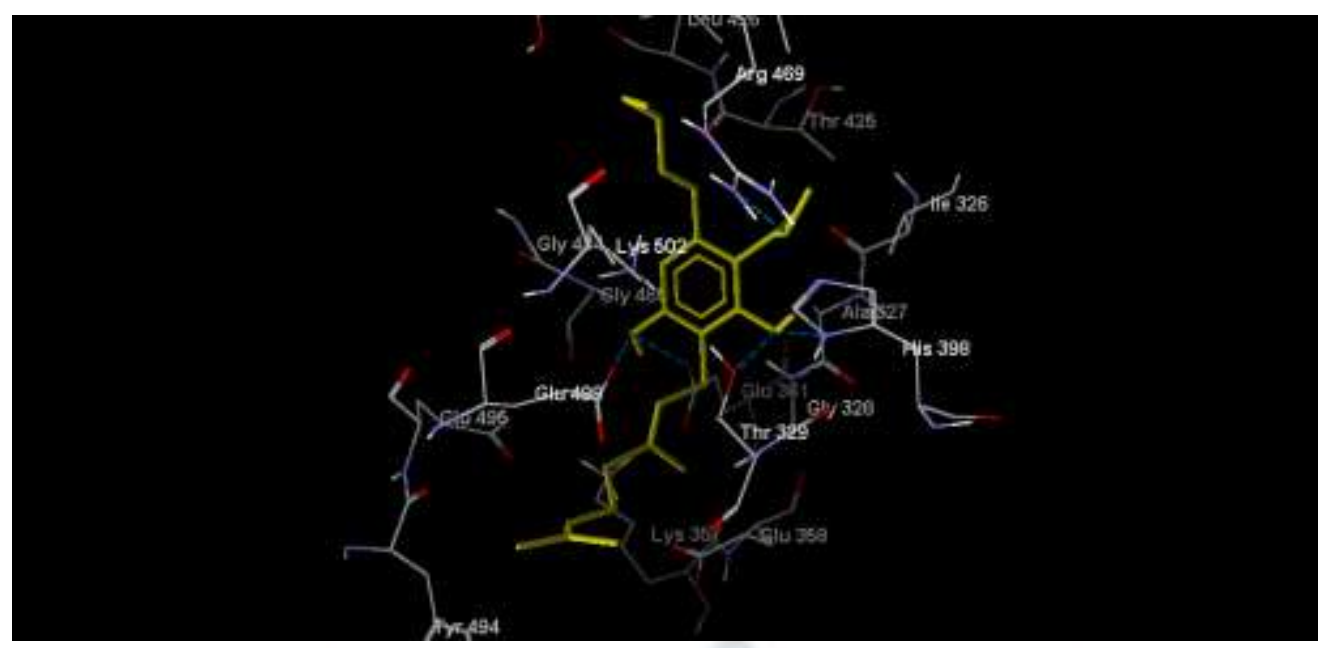

Fig 7a. Docking of molecule 4 pose 2644999 like compound from zinc Interacting residues are Gly 485, glu 498, glu 361, arg 469, thr 329 and his 398

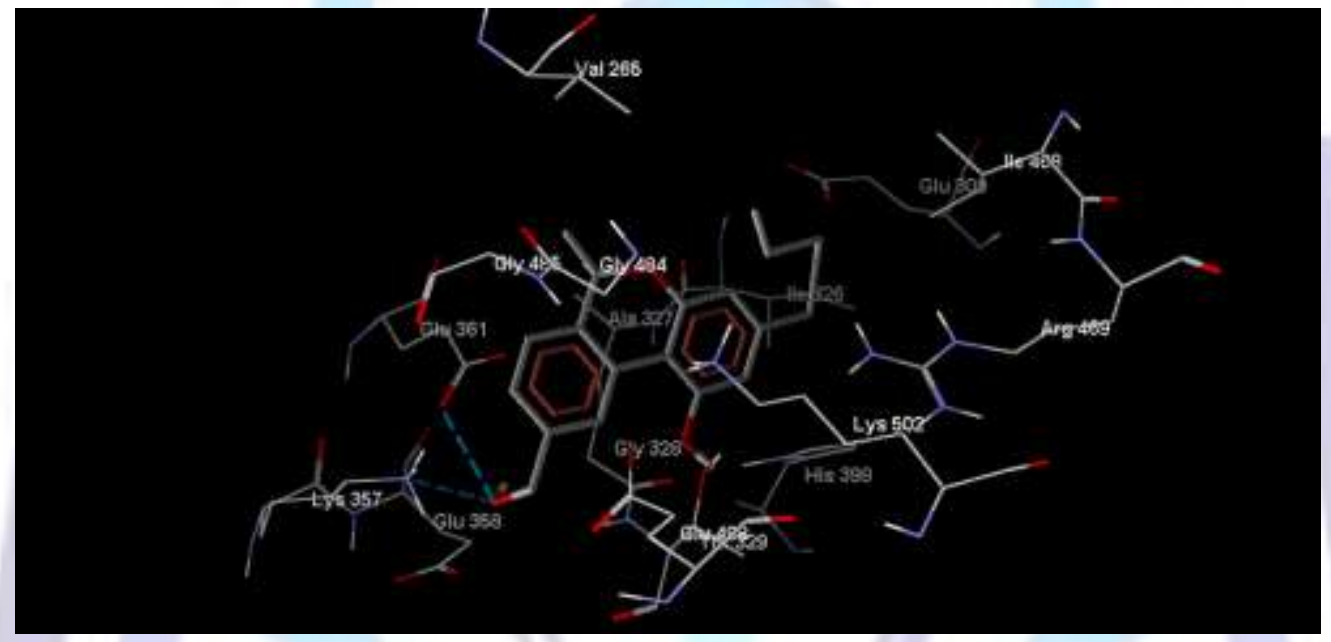

Fig 6b: Docking of molecule17 pose 4 of 3081990 like compound from zinc

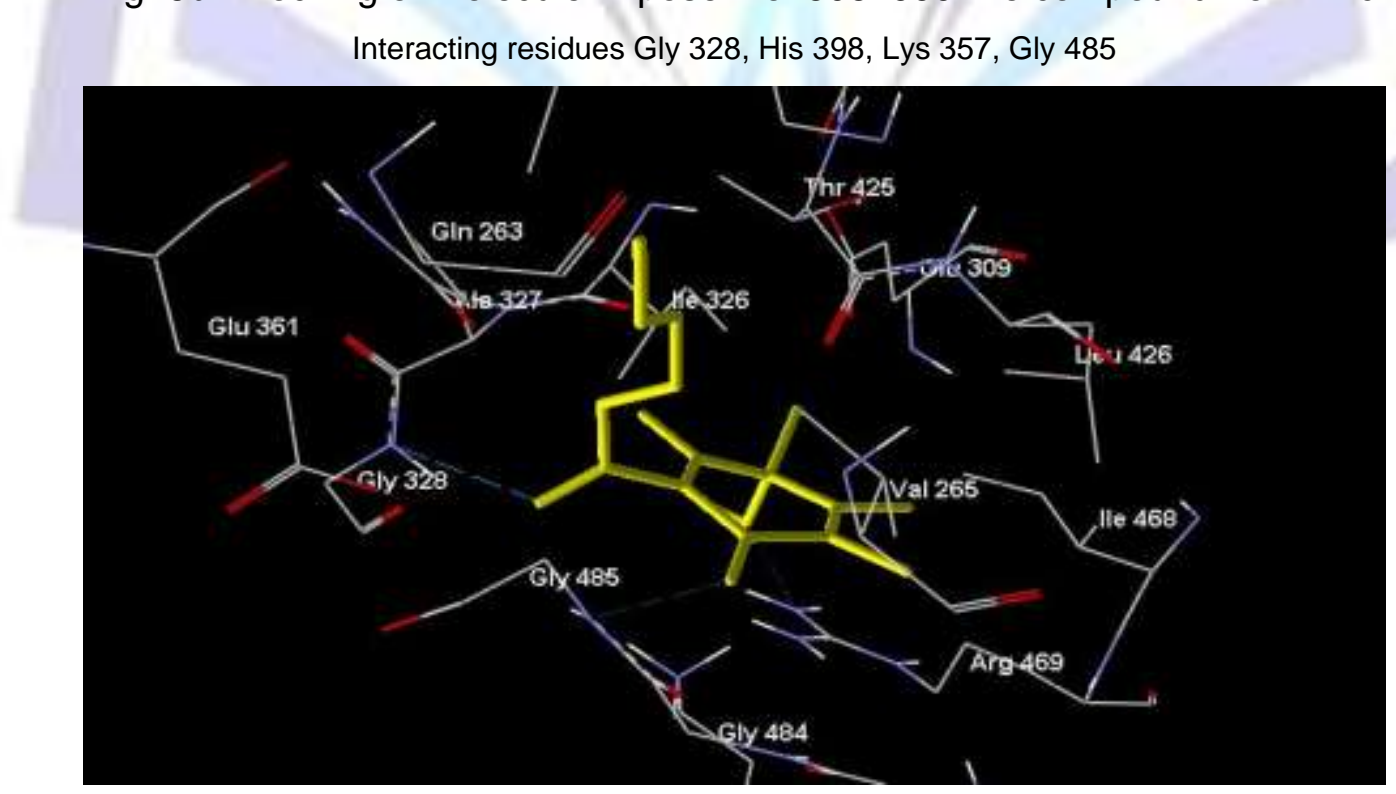

Fig 6c: Docking of molecule 2 pose 7 of 193681 like compound from zinc. Interacting residues Gly 328,Gly 485,Arg 469 


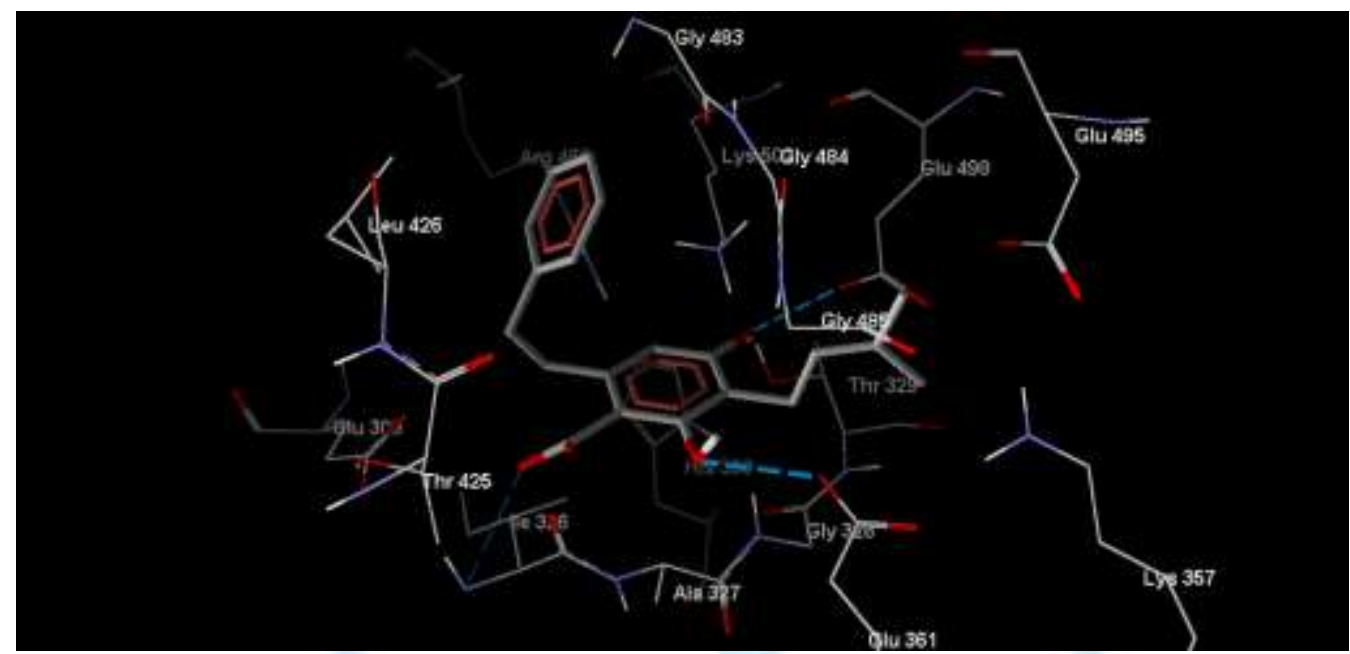

Fig 6d. Docking of molecule 17 pose 1 of 644999 like compound from zinc Interacting residues lle 326, Glu 361, Glu 498.

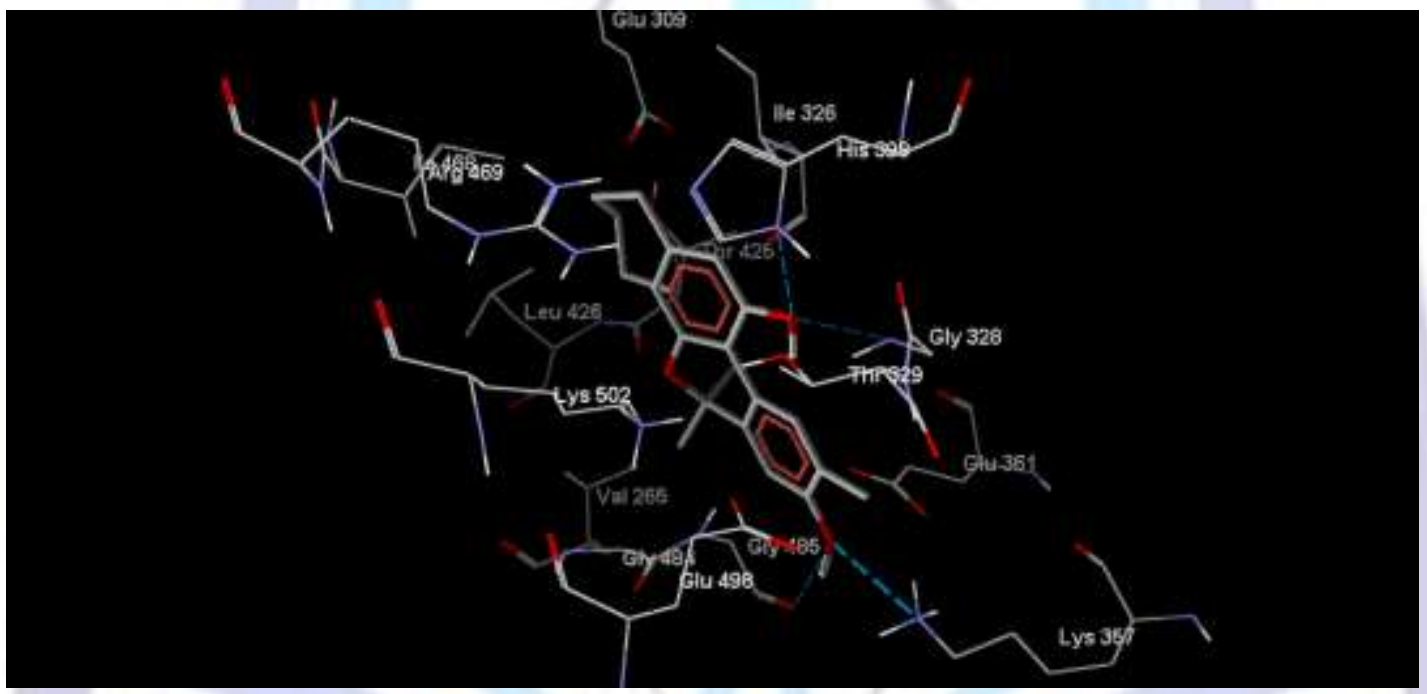

Fig 6e: Docking of molecule15 pose 5 of 3081990 like compound from zinc

Interacting residues Gly 328, His 398, Lys 357, Gly 485

Bioinformatics involves discovery, development and implementation of computational algorithms and software tools that facilitate an understanding of the biological processes with the goal to serve primarily healthcare sectors with several spin-offs. It is well-known that the key enzymes involved in the vital bio-chemical pathways of bacteria are potential targets for antibacterial drugs. The tryptophan biosynthetic pathway is a crucial metabolic pathway for the survival of M. tuberculosis (Parish and Stoker 2002; Sassetti et al., 2003), and meanwhile does not exist in human (Xie et al., 2003). Thus, key enzymes involved in this pathway are potential drug targets for new anti-TB drugs.

The purpose of this study is to identify the binding interaction having more binding energy towards the MTB AS1. It is showed from the result of multiple sequence alignment (Clustal $X$ ) between template ( $S$. marcescens) and query (AS1 of $\mathrm{mtb}$ ) that the alignment score was 481 . The objective of this project is to generate the protein AS1 of $\mathrm{mtb}$ and drugs targeted at specific docking of protein with inhibitor solution of the structure of MTB AS1 protein has led to the design of novel small molecule inhibitors. From the $20 \mathrm{pdb}$ structures generated by the MODELLER we have selected least objective function structure for better inhibitory results. This structure was submitted to Ramachandran plot and it was identified that the generated model was reliable. This 3D structure was again improved through Molecular dynamics studies and finally active site residues were identified using Castp server. The residues, GLU 348, GLY 475 GLY 324 THR 325 GLU 488 LYS 492 ILE 322 THR 415 GLU 303 were involved in active site of the MTB AS1. According to data presented in results, it was observed that drugs inhibited AS1 of $\mathrm{mtb}$. As mentioned by the data above, it suggests form testing of these drugs at experimental 
investigation. There is now great excitement in the field of TB research with the development of a MTB AS1 protein inhibitor.

\section{REFERENCES}

1. E.L.Corbett, C.J.Watt, N.Walker, D.Maher, B.G.Williams, M.C. Raviglione,C. Dye, The growing burden of tuberculosis: global trends and interactions with The HIV epidemic, Arch. Intern. Med.163 (2003)10091021.

2. World Health Organization, Anti-tuberculosis drug resistance in the world: The Global Report (2004).

3. T.Parish, N.G. Stoker, The common aromatic amino acid biosynthesis pathway is essential in Mycobacterium tuberculosis, Microbiology148 (2002) 3069-3077.

4. C.M. Sassetti, D.H. Boyd, E.J. Rubin, Genes required for myco bacterial growth defined by high density mutagenesis, Mol. Microbiol.48 (2003)77-84.

5. G.Xie, N.O. Keyhani, C.A. Bonner, R.A. Jensen, Ancient origin of the tryptophan operon and the dynamics of evolutionary change, Microbiol. Mol. Biol.R. 67 (2003) 303-342.

6. A.A.Morollo, M.J.Eck, Structure of the cooperative allosteric anthranilate synthase from Salmonella typhimurium, Nat. Struct.Biol.8 (2001) 243-247.

7. G.Spraggon, C.Kim, X.Nguyen-Huu, M.-C.Yee, C.Yanofsky, S.E.Mills, The Structures of anthranilate synthase of Serratia marcescens crystallized in the Presence of (i) its substrates, chorismate and glutamine, and a product, glutamate, and (ii) its end-product inhibitor, L-tryptophan, PNAS98 (2001) 6021-6026.

8. T. Knochel, A.Ivens, G.Hester, A. Gonzalez, R. Bauerle, M. Wilmanns, K. Kirschner, J.N. Jansnius,The crystal structure of anthranilate synthase from Sulfolobus solfataricus: Functional implications (X-ray structure analysisy/ Glutamine amido transferase y/ tryptophan biosynthesis), Proc. Natl. Acad. Sci. USA96 (1999)9479-9484.

9. H.Zalkin, D.Kling, Anthranilate synthetase:purification and properties of component I from Salmonella typhimurium, Biochemistry 7 (1968) 3566-3573.

10. J.Ito,E.C.Cox,C.Yanofsky, Anthranilate synthetase, an enzyme specified by the tryptophan operon of Escherichia coli: purification and characterization of component I,J. Bacteriol.97(1969) 725-733.

11. C.N. Hankins, S.E. Mills, Anthranilate synthase-amidotransferase (combined). A novel from ofantrhanilate synthase from Euglena gracilis, J. Biol. Chem.251 (1976)7774-7778

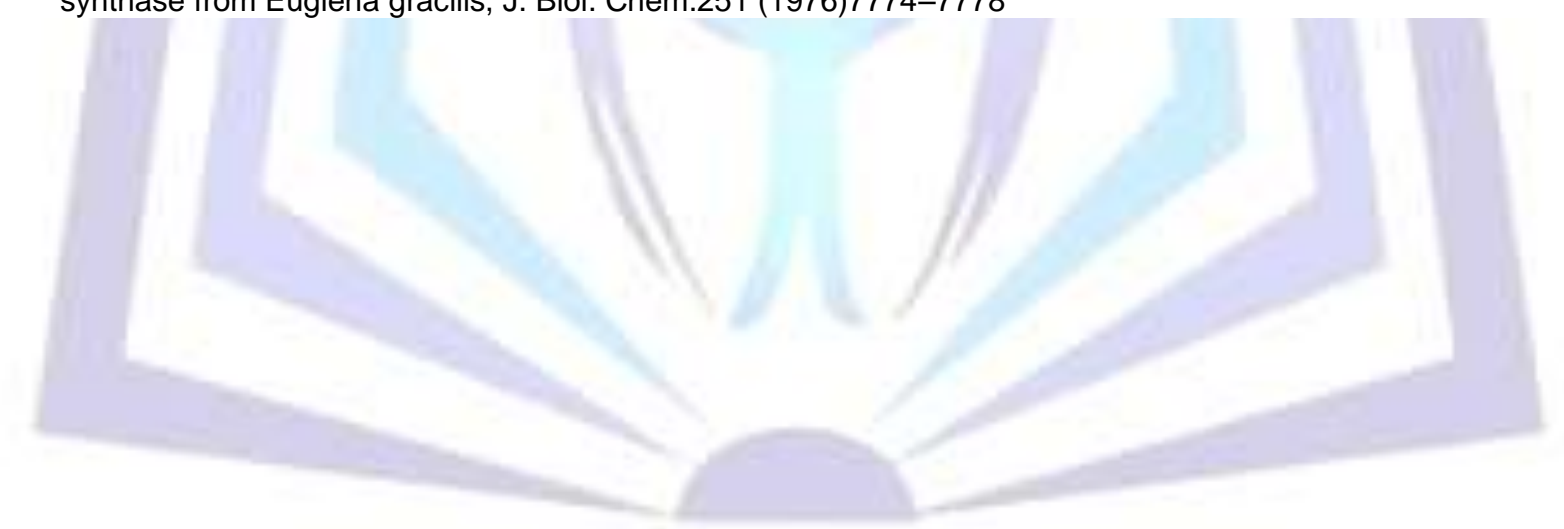

